\title{
Características clínico-epidemiológicas de pacientes de COVID-19 en una Unidad de Medicina Familiar en Reynosa,Tamaulipas
}

\section{Clinical-epidemiological characteristics of COVID-19 patients in a Family Medi- cine Unit in Reynosa, Tamaulipas}

\author{
Verónica Martínez-Lara', Dionicio Morales-Ramírez², Mayra Vázquez-Meza ${ }^{3}$
}

1. Médico Familiar, adscrito al Instituto Mexicano del Seguro Social, Unidad de Medicina Familiar No. 77, atención primaria, Ciudad Madero, Tamaulipas, México. Orcid: https://orcid.org/0000-0002-9338-4845

2. Doctor en Ciencias Sociales con orientación en desarrollo sustentable, adscrito a la Facultad de Ingeniería "Arturo Narro Siller" de la Universidad Autónoma de Tamaulipas, Profesor-investigador. Tampico, Tamaulipas, México. Orcid: https://orcid.org/0000-0002-6859-6547

3. Médico Familiar, adscrito al Instituto Mexicano del Seguro Social, Unidad de Medicina Familiar No. 7, jefa de departamento clínico, Reynosa, Tamaulipas. Orcid: https://orcid.org/0000-0003-0953-6728

\footnotetext{
*Autor de correspondencia: Dionicio Morales-Ramírez

Universidad Autónoma de Tamaulipas, Centro Universitario Tampico-Madero, Tamaulipas.

Teléfono: (55) 4811119863 Correo electrónico: dionicio.morales@gmail.com
}

DOI http://dx.doi.org/10.28960/revmeduas.2007-8013.v12.n1.003

Recibido 07 de septiembre 2021, aceptado 29 de octubre 2021

RESUMEN

Objetivo: Identificar las características clínico-epidemiológicas que presentan los pacientes que se atendieron por presentar síntomas de COVID-19 en la UMF No 40 del Instituto Mexicano del Seguro Social situado en Reynosa, Tamaulipas. Material y métodos: Se llevó a cabo un estudio observacional, transversal y descriptivo, empleando información recogida en el formato emitido por la Secretaría de Salud de México en un total de 323 pacientes sospechosos. Además, se estimaron pruebas de asociación y diferencia de medias. Resultados: Se encontró que, del total de encuestados, el 53.9\% fue diagnosticado como positivo mediante la prueba Reacción de Cadena de Polimerasa (PCR). La comorbilidad que más se presentó en este grupo fue la obesidad (32.8\%), seguida de la hipertensión (23\%) y la diabetes (17.8\%). Los hallazgos de las pruebas de asociación indican que no existe una relación entre poseer determinada comorbilidad y dar positivo a la prueba. En tanto que la prueba de diferencia de medias señala que el número de síntomas entre los pacientes positivos suele ser mayor respecto de los negativos. Y se confirma que las personas con mayor edad suelen estar más predispuestos a ser contagiados respecto de los más jóvenes. Conclusiones: Cada población responde de manera distinta a una enfermedad, por ello, los estudios epidemiológicos nos ayudan a entender el comportamiento de una patología en un contexto especifico, por lo cual, es necesario continuar generando estudios a nivel local.

Palabras claves: Covid 19, prueba t, comorbilidades, sintomatología.

\begin{abstract}
Objective: Identify the clinical-epidemiological characteristics of the patients who presented symptoms of COVID-19 at the UMF No 40 of the Instituto Mexicano del Seguro Social located in Reynosa, Tamaulipas. Material and methods: An observational, retrospective, cross-sectional, descriptive, retrospective study was carried out, using information collected in the format issued by the Mexican Ministry of Health in a total of 323 suspected patients. In addition, tests of association and mean difference were estimated. Results: It was found that, of the total respondents, $53.9 \%$ were diagnosed as positive by the Polymerase Chain Reaction (PCR) test. The most common comorbidity in this group was obesity $(32.8 \%)$, followed by hypertension (23\%) and diabetes $(17.8 \%)$. The findings of the tests of association indicate that there is no relationship between having a certain comorbidity and testing positive. Meanwhile, the mean difference test indicates that the number of symptoms among positive patients tends to be greater than among negative patients. And it is confirmed that older people tend to be more likely to be infected than younger people. Conclusions: Each population responds differently to a disease; therefore, epidemiological studies help us to understand the behavior of a pathology in a specific context, which is why it is necessary to continue generating studies at the local level.
\end{abstract}

Key words: Covid 19, t-test, comorbidities, symptomatology.

\section{Introducción}

Las enfermedades infecciosas han estado pre-

sentes a lo largo de la historia de la humanidad, aunque en los últimos años han aparecido más y nuevas de diversa etiología, las cuales han sido capaces de cambiar los patrones de morbilidad y mortalidad, al grado de convertirse en pandemias ${ }^{1}$. 
La pandemia más reciente declarada por la Organización Mundial de la Salud fue en el 2020, la cual fue causada por un virus que afecta las vías respiratorias de manera similar a la neumonía. Dicha enfermedad fue denominada coronavirus desease 2019 (COVID-19), así como coronavirus-2 del síndrome respiratorio agudo grave (SARS-CoV-2) ${ }^{2}$. Su propagación suele ser de forma directa de persona a persona a través de gotículas provenientes de la nariz o de la boca de individuos infectados. $O$ de manera indirecta, cuando algún infectado estornuda o habla y deja gotas con el virus sobre una superficie $^{2}$. Es importante mencionar que dicho virus puede sobrevivir de 4 hasta 72 horas en la superficie dependiendo del material que esta sea. De ahí la importancia de las medidas preventivas como el uso de cubrebocas o el lavado continuo de manos con agua y jabón ${ }^{3}$.

Aunque sigue sin existir una explicación definitiva sobre el origen de este virus y sobre un tratamiento o vacuna que inmunice a las personas. La enfermedad ha demostrado una gran diversidad de síntomas como fiebre, tos, anosmia/disgeusia, fatiga, disnea, síntomas gastrointestinales, mialgias, conjuntivitis, odinofagia, cefalea y rinorrea entre otras ${ }^{4-12}$ así como una prevalencia en personas con problemas de obesidad, hipertensión arterial y diabetes entre otras comorbilidades $5,10,13$.

En México, las estadísticas a nivel nacional publicadas en la página COVID-19 México del Go- bierno Federal al día 12 de febrero del 2021, reportan $1,968,566$ casos confirmados como positivos, de los cuales, el $50.12 \%$ son hombres, el $49.88 \%$ son mujeres. El $17.46 \%$ tiene problemas de hipertensión, el $14.76 \%$ de obesidad, el $13.46 \%$ de diabetes y el $7.39 \%$ de tabaquismo ${ }^{14}$. Sin duda, la prevalencia de comorbilidades y la aparición de sintomatología puede variar de región en región, de ahí que los estudios que ayuden a brindar información sobre ambos temas contribuyen a entender mejor dicha enfermedad.

Así por ejemplo en el Hospital Civil de Culiacán, en una muestra de 192 pacientes confirmados se encontró que la obesidad (33.3\%), hipertensión arterial (31.7\%) y diabetes mellitus (21.9\%) fueron las principales comorbilidades, en tanto que la clínica más frecuente fue la fiebre $(84.4 \%)$, tos $(83.8 \%)$, cefalea $(74.8 \%)$ y disnea (65.1\%). La letalidad en esta muestra fue del $31.25 \%{ }^{5}$. Otro trabajo realizado en un en un hospital de tercer nivel en la ciudad de México, se empleó una muestra de 209 pacientes diagnosticados por COVID-19 concluyendo que la edad promedio de los infectados fue de 43 años con una concentración en los rangos de 33 a 54 años, siendo hombres con obesidad y sobrepeso los más frecuentes. Asimismo, se encontró que los adultos mayores con comorbilidades que presentaron fiebre, tos y disnea fueron más propensos a ser admitidos en el hospital para su tratamiento. En tanto que los pacientes internados con historial de diabetes, alto índice de 
masa corporal, así como laboratorios compatibles con estado inflamatorio fueron más propensos a requerir cuidados intensivos ${ }^{15}$.

A pesar de los esfuerzos que se han hecho, la detección de la enfermedad aun no puede ser diagnosticada de manera precisa mediante un cuadro clínico o definición operacional, por lo que el uso de pruebas de laboratorio como la prueba Reacción en Cadena de la Polimerasa (PCR) son necesarias para confirmar o descartar si una persona detectada como sospechosa es positiva a la enfermedad. Por tal motivo, en este trabajo se lleva a cabo una identificación de las características clínico-epidemiológicas que presenta la muestra de pacientes de manera general, así como separada en dos grupos, los positivos y negativos a la prueba PCR por COVID-19.

\section{Material y métodos}

Estudio observacional, transversal y descriptivo, el cual, tiene como propósito explorar las características de los pacientes con diagnóstico de caso sospechoso de COVID-19 en la Unidad de Medicina Familiar No. 40 del Instituto Mexicano del Seguro Social, situada en Reynosa, Tamaulipas. A los cuales se les práctico la prueba de Reacción de Cadena de Polimerasa (PCR) para confirmar o descartar la presencia de dicha enfermedad.

El instrumento y la muestra: Los datos empleados en este trabajo fueron generados a partir de la encuesta emitida por la Secretaria de Salud de México del 22 de marzo al 12 de septiembre del 2020 comprendiendo 24 semanas epidemiológicas de la 13 a la 37 . El cual fue aplicado por el departamento de epidemiologia de la UMF 40. Después de revisar la información se decidió eliminar a los casos sospechosos que no contaban con los resultados de la prueba PCR por lo que la muestra quedó conformada por 323 pacientes.

Análisis de los datos: El análisis de la información se llevó a cabo mediante el software SPSS versión 20 en tres pasos. En primer lugar, se estimaron las estadísticas básicas, así como gráficas sobre las comorbilidades y la sintomatología o clínica que presenta la muestra de forma general. En segundo lugar, debido al alto número de comorbilidades registradas en el cuestionario, se procedió a elaborar el índice de sintomatología, el cual está integrado por los 20 síntomas de la gráfica 1 y en donde cada una de ellas es una variable que toma el valor de 1 si el paciente presenta dicho síntoma y cero si no lo presenta. Después se suma el valor de cada variable y el resultado se estandariza mediante el criterio de MIN-MAX para asegurarnos que el resultado oscile entre 0 y 1 . De tal manera que, los valores cercanos a 1 expresen una mayor presencia de síntomas y valores cercanos a cero, su ausencia. Con esta nueva variable se lleva cabo la estimación de la prueba t para muestras independientes afín de determinar si existen diferencias estadísticas entre el 
número de síntomas entre los pacientes positivos y negativos a la prueba PCR. También se aplica esta prueba para identificar si existen diferencias entre el sexo de las personas y su edad, sobre el diagnostico positivo o negativo de la PCR. Por último, se lleva cabo un análisis de asociación mediante el Chi Cuadrado de Pearson para identificar la existencia de alguna relación entre poseer determinada comorbilidad y el resultado de la prueba PCR.

\section{Resultados}

Se identificaron 323 pacientes sospechosos, de los cuales, el $52.9 \%$ pertenece al sexo femenino y $47.1 \%$ al masculino. La edad promedio estimada fue de 40 años y se encontró que el 29.7\% dijo ser trabajador del Instituto Mexicano del Seguro Social (IMSS). Del total de pacientes encuestados, el $53.9 \%$ fue positivo y el $46.1 \%$ negativo. Una vez que se identificó en la muestra los casos positivos, se identificó que los hombres son los que presentaron una mayor frecuencia de contagio con $50.6 \%$, en tanto que, en las mujeres fue de $49.4 \%$. La edad promedio registrada fue de 42 años en los casos positivos y de 37.9 años en los negativos. Respecto a los síntomas se encontró de manera general en toda la muestra la siguiente distribución (Véase Figura 1).
Figura 1. Sintomatología en casos sospechosos

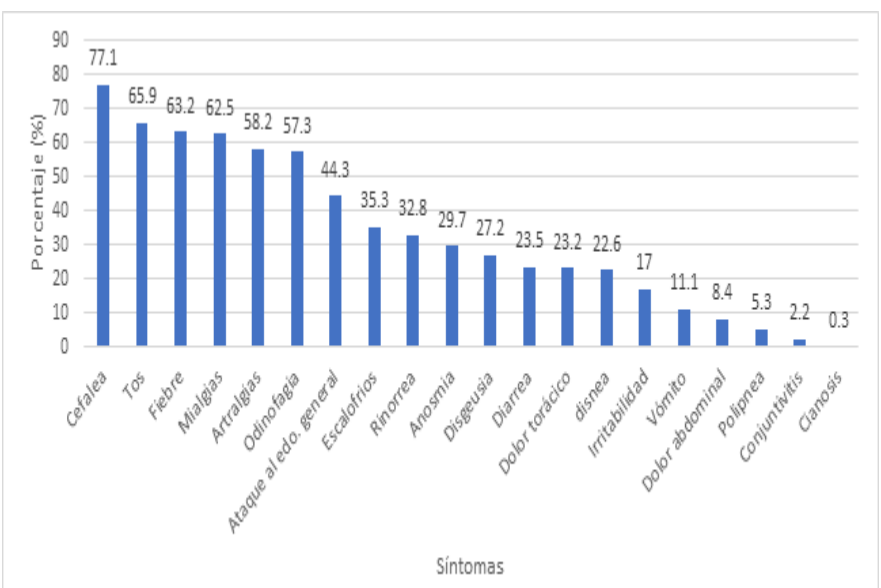

Fuente: elaboración propia.

La figura 1 muestra de manera general la distribución de la sintomatología de los casos sospechosos, en donde la cefalea, la tos y la fiebre fueron las principales. Lo cual, refleja la definición operacional para la vigilancia epidemiológica de enfermedad respiratoria viral ${ }^{16}$.

Para visualizar de mejor manera la distribución de los síntomas en los pacientes, se llevó a cabo una separación entre los casos positivos y negativos diagnosticados mediante la prueba PCR. En la Tabla 1, se puede aprecia que la cefalea ocupa la primera posición dentro de los síntomas más recurrentes con un $73 \%$ en los casos positivos, en tanto que la polipnea fue la de menor frecuencia con $6.9 \%$. 
Tabla 1. Síntomas frecuentes en los casos positivos

\begin{tabular}{clc}
\hline Ranking & \multicolumn{1}{c}{ Sintomatología } & $\begin{array}{c}\text { Casos Positivos } \\
\text { Sí (\%) }\end{array}$ \\
\hline 1 & Cefalea & 73.0 \\
2 & Tos & 64.9 \\
3 & Mialgias & 64.4 \\
4 & Artralgias & 60.9 \\
5 & Fiebre & 60.3 \\
6 & Odinofagia & 55.7 \\
7 & Ataque al estado general & 49.4 \\
8 & Rinorrea & 39.1 \\
9 & Escalofríos & 36.8 \\
10 & Anosmia & 29.9 \\
11 & Disgeusia & 28.2 \\
12 & Dolor torácico & 24.1 \\
13 & Diarrea & 23.0 \\
14 & Disnea & 22.4 \\
15 & Irritabilidad & 19.0 \\
16 & Vómito & 9.2 \\
17 & Polipnea & 6.9 \\
18 & Dolor abdominal & 6.9 \\
19 & Conjuntivitis & 2.3 \\
20 & Cianosis & 0.6 \\
\hline
\end{tabular}

Fuente: Elaboración propia

En el caso de los pacientes sospechosos que dieron negativo a la prueba, la sintomatología tuvo diferente distribución. En ambas tablas la cefalea y la tos ocuparon las primeras dos posiciones, pero a partir de la tercera posición la frecuencia en las sintomatologías son diferentes (Tabla 2).

Afín de identificar si en promedio el número de síntomas es el mismo entre ambos grupos, se llevó a cabo una prueba de hipótesis sobre la media de ambos grupos. Para lo cual, se elaboró el índice de sintomatología, tal y como se mencionó en la sección de la metodología. En el caso de los pacientes negativos dicho índice fue de 0.392 en tanto que para los positivos fue de 0.517 . Mediante la prueba t para muestras independientes se encontró con un nivel de significancia del $95 \%$ que la media de ambos grupos es diferente ( $p$-valor 0.000 ). Por lo tanto, podemos afirmar que los pacientes positivos tienden a presentar mayores síntomas respecto de los que son negativos. De la misma forma, se llevó a cabo la identificación de las comorbilidades en la muestra sobre los casos sospechosos. Véase la Figura 2.

Tabla 2. Síntomas frecuentes en los casos negativos

\begin{tabular}{llc}
\hline Ranking & Sintomatología & $\begin{array}{c}\text { Casos } \\
\text { Negativos } \\
\text { Sí (\%) }\end{array}$ \\
\hline 1 & Cefalea & 81.9 \\
2 & Tos & 67.1 \\
3 & Fiebre & 66.4 \\
4 & Mialgias & 60.4 \\
5 & Odinofagia & 59.1 \\
6 & Artralgias & 55.0 \\
7 & Ataque al estado ge- & 38.3 \\
8 & neral & 33.6 \\
9 & Escalofríos & 29.5 \\
10 & Anosmia & 26.2 \\
11 & Disgeusia & 25.5 \\
12 & Rinorrea & 24.2 \\
13 & Diarrea & 22.8 \\
14 & Disnea & 22.1 \\
15 & Dolor torácico & 14.8 \\
16 & Irritabilidad & 13.4 \\
17 & Dómito & 10.1 \\
18 & Dolor abdominal & 3.4 \\
19 & Polipnea & 2.0 \\
20 & Conjuntivitis & 0.0
\end{tabular}

Fuente: Elaboración propia 
Figura 2. Frecuencia de comorbilidades en casos sospechosos

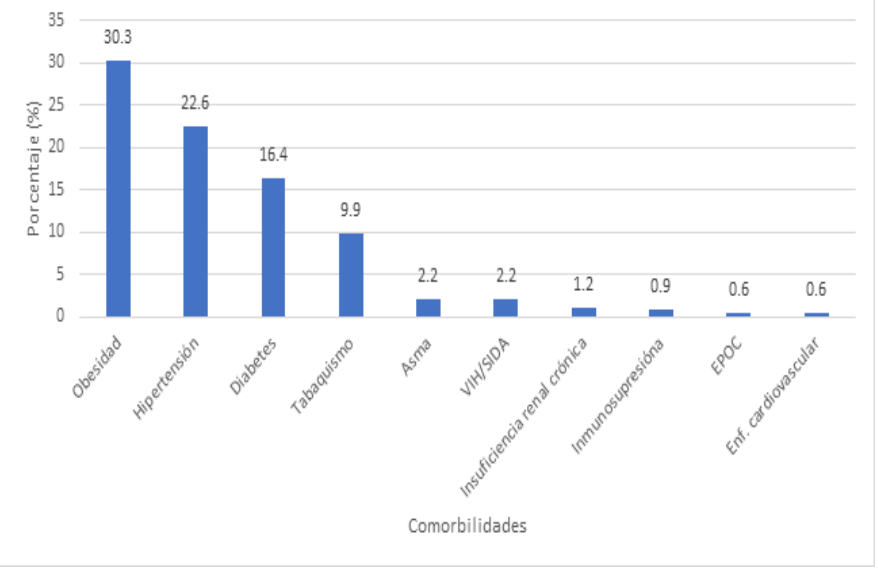

Fuente: elaboración propia.

Se observa que la obesidad, la hipertensión y la diabetes son las tres principales comorbilidades. Al realizar la distribución de frecuencias controlada por grupos los resultados se mantuvieron para las primeras cuatro comorbilidades. Aunque en los casos positivos los valores porcentuales fueron mayores en las primeras tres.

Tabla 3. Distribución porcentual de comorbilidades por grupo

\begin{tabular}{lcccc}
\hline Comorbilidad & $\begin{array}{c}\text { Caso } \\
\text { Positi- } \\
\text { vos } \\
(\%)\end{array}$ & $\begin{array}{c}\text { Ran- } \\
\text { king }\end{array}$ & $\begin{array}{c}\text { Caso } \\
\text { Negativo } \\
(\%)\end{array}$ & Ranking \\
\hline Obesidad & 32.8 & 1 & 27.5 & 1 \\
Hipertensión & 23.0 & 2 & 22.1 & 2 \\
$\begin{array}{l}\text { Diabetes } \\
\text { Tabaquismo }\end{array}$ & 17.8 & 3 & 14.8 & 3 \\
$\begin{array}{l}\text { VIH/SIDA } \\
\text { Insuficiencia re- }\end{array}$ & 1.2 & 4 & 10.7 & 4 \\
nal crónica & 1.7 & 5 & 2.7 & 6 \\
$\begin{array}{l}\text { EPOC } \\
\text { Asma }\end{array}$ & 1.1 & 7 & 0.7 & 9 \\
$\begin{array}{l}\text { Inmunosupre- } \\
\text { sión }\end{array}$ & 1.1 & 8 & 3.4 & 5 \\
$\begin{array}{l}\text { Enfermedad car- } \\
\text { diovascular }\end{array}$ & 0.6 & 9 & 1.3 & 7 \\
\hline Fuente: elaboracionn & 0.6 & 10 & 0.7 & 8 \\
\hline
\end{tabular}

Fuente: elaboración propia
Adicionalmente se hicieron pruebas de asociación entre variables de tipo cualitativo como la presencia o ausencia de comorbilidades y el resultado de la prueba PCR (positivo o negativo). Así como, el sexo de la persona y resultado de la prueba PCR (positivo o negativo). Ambas relaciones fueron estimadas mediante la prueba del Chi Cuadrado de Pearson. En todos los casos no se encontró evidencia de una asociación entre poseer algún tipo de comorbilidad y dar positivo o negativo a la prueba de PCR o ser hombre o mujer y dar positivo o negativo a la enfermedad. Por lo que no hay evidencia estadística de que poseer determinada comorbilidad o sexo, te haga más propenso al contagio del virus SARS-COV-2.

Por último, se calculó el promedio de edad entre los dos grupos y se encontró que la media de los pacientes positivos fue de 42 años, en tanto que la media de los pacientes negativos a la prueba fue de 37.9 años. Con esta información se estimó la prueba t para muestras independientes con el fin de identificar si la media de ambos grupos es estadísticamente diferente entre sí. Los resultados de la prueba señalan con un nivel de significancia del $95 \%$ que la media de ambos grupos es diferente ( $p$-valor 0.002). Por lo tanto, podemos decir que la edad promedio del grupo de los casos positivos es mayor respecto de la edad de los casos negativos. 


\section{Discusión}

La pandemia actual es ocasionada por un virus nuevo para la humanidad conocido como SARCOV-2, del cual, se conoce muy poco. Aunque de manera paulatina se ha obtenido conocimiento de sus características clínicas y epidemiológicas, su manejo y tratamiento aún no han logrado controlar o disminuir los contagios. La causa de que la tasa de casos positivos se esté elevando es atribuida a diversos factores, entre ellos el desconocimiento de la evolución natural de la enfermedad por lo que es necesario identificar las características clínico-epidemiológicas de los pacientes de cada lugar.

Durante el periodo de tiempo analizado, se ha identificado la presencia de cuatro comorbilidades presentes en los casos positivos, sin embargo, su comportamiento o frecuencia entre países y regiones suele variar, ello debido a que en cada lugar las condiciones físicas, sociales, culturales, económicas y ambientales son diferentes, favoreciendo la presencia o ausencia de ellas. Así, por ejemplo, en la muestra analizada se encontró que la obesidad es la principal comorbilidad con un $32.8 \%$, seguida de la hipertensión arterial con un 23\%. En tercer lugar, la diabetes mellitus tipo 2 con $17.8 \%$ y en el tabaquismo $9.2 \%$. Dichos valores siguen una distribución muy parecida a las reportadas en el Hospital Civil de Culiacán, aunque los valores suelen ser más altos en los segundos 5 . Al parecer la obesidad es la principal comorbilidad, lo cual puede ser un reflejo de un estilo de un mal estilo de vida, caracterizado por una mala alimentación y falta de actividades físicas. Vale la pena comentar que a nivel nacional el principal problema es hipertensión, seguida de la obesidad y la diabetes mellitus en casos positivos. ${ }^{14}$ Asimismo, los resultados de este análisis señalan que, dentro de los casos positivos, los hombres fueron los más frecuentes $(50.6 \%)$ resultado muy similar a los valores nacional y al de otras clínicas en el país. ${ }^{5,15}$

En cuestión de la sintomatología o clínica más frecuente, en la UMF 40 de Reynosa Tamaulipas, se encontró que, en los casos positivos, la cefalea, tos, mialgias, artralgias y fiebre, son los cinco principales síntomas, contrastando con el caso del Hospital Civil de Culiacán en donde la fiebre fue la principal clínica, seguida de la tos, cefalea y disnea. ${ }^{5}$ Confirmándose que tanto la presencia de las comorbilidades como de la sintomatología depende de las características de cada región o área de estudio.

Además, se llevó a cabo una prueba de hipótesis para identificar si el número de síntomas que presentan los casos positivos versus los negativos en la muestra de análisis son distintos entre ellos. Los resultados de la prueba t para muestras independientes señala que el número de síntomas entre ambos casos es diferente. Por lo tanto, podemos afirmar que los pacientes positivos tienden a presentar mayores síntomas respecto de los que son negativos. 


\section{Conclusiones}

Cada población responde de manera distinta a una enfermedad, siendo los estudios epidemiológicos los que nos ayudan a entender el comportamiento de una patología en un contexto especifico, por lo cual, es necesario continuar generando estudios a nivel local. De manera general se encontró que los hombres de 42 años en promedio fueron los que dieron positivo a la prueba PCR, sin embargo, ello no sugiere que el género de las personas se encuentre asociado de alguna forma al riesgo de contagio, tal y como se demostró. Referente a las principales comorbilidades se destaca la obesidad y la hipertensión, en tanto que la cefalea, la tos y las mialgias son las clínicas más frecuentes en los casos positivos en esta muestra de análisis. Además, se demostró que el riesgo de contagio no está asociado algún tipo de comorbilidad. Esto, no significa que las personas con algún tipo de comorbilidad no estén en riesgo y no deban de cuidarse, lo que el estudio sugiere es que las comorbilidades seguramente están asociadas al riesgo de complicación de la enfermedad, más que del contagio. Por último, se comprobó que la edad promedio del grupo de personas positivas o contagiadas, es mayor respecto de la edad de los casos negativos. Sugiriendo que las personas adultas son más propensas al contagio.

\section{Agradecimiento}

Se agradece al director de la Unidad de Medicina Familiar No 40 de Reynosa, Tamaulipas, el Dr. Francisco Emmanuel Díaz Cisneros por brindar su autorización y visto bueno para llevar a cabo este trabajo. Así como al departamento de epidemiología de la misma unidad por brindar el acceso a la información estadística.

\section{Referencias}

1. Villamil-Jiménez LC. Epidemias y pandemias: una realidad para el siglo XXI. Un mundo y una salud. Rev. Lasallista Investig. 2013; 10(1): 7-8.

2. Organización Mundial de Salud. Preguntas frecuentes sobre los nuevos coronavirus. [Internet]. [Consultado 12 agosto 2020]. OMS; 2020. Disponible en: https://www.who.int/es/emergencies/diseases/novel-coronavirus-2019/question-andanswers-hub/q-a-detail/q-a-coronaviruses

3. World Health Organization. Tiempo que sobrevive el virus en las superficies. [Consultado 26 de octubre de 2020] Disponible en el sitio web: https://www.who.int/es/emergencies/diseases/novel-coronavirus-2019/advice-for-public/q-a-coronaviruses.

4. Madrigal-Rojas JP, Quesada-Loría M, García-Sánchez M, Solano-Chinchilla A. SARS CoV-2, manifestaciones clínicas y consideraciones en el abordaje diagnóstico de $\mathrm{CO}$ VID- 19. Rev Méd Costa Rica. 2020; 85 (629): 13-21. 
5. Villagrán-Olivas K, Torrontegui-Zazueta L, Entzana-Galindo A. Características clínicoepidemiológicas de pacientes de COVID-19 en un Hospital de Sinaloa, México. Rev Med UAS. 2020;10 (2): 65-79.

6. Guan Wj, Ni Zy, Hu Y, Liang Wh, Ou Cq, He $\mathrm{Jx}$, et al. Clinical characteristics of coronavirus disease 2019 in China. N Engl $\mathrm{J}$ Med. 2020;58 (4):711-712.

7. Huang C, Wang Y, Li X, Ren L, Zhao J, Hu $Y$, et al. Clinical features of patients infected with 2019 novel coronavirus in Wuhan, China. Lancet. 2020; 395 (10223):497-506.

8. Cheung KS, Hung IFN, Chan PPY, Lung KC, Tso E, Liu R, et al. Gastrointestinal manifestations of SARS-CoV-2 infection and virus load in fecal samples from the hong kong cohort and systematic review and meta-analysis. Gastroenterology. 2020; 159 (1): 81-95.

9. Lechien JR, Chiesa-Estomba CM, De Siati DR, Horoi M, Le Bon SD, Rodriguez A, et al. Olfactory and gustatory dysfunctions as a clinical presentation of mild-to-moderate forms of the coronavirus disease (COVID19): a multicenter European study. Eur Arch Otorhinolaryngol. 2020;277 (8): 2251-226.

10. Díaz-Castrillón FJ, Toro-Montoya AI. SARSCoV-2/COVID-19: The virus, the disease and the pandemic. Med \& Lab. 2020; 24 (3):183-205.

11. Graham CW, Dela-Cruz C.S, Cao B, Pasnick S, Jamil S. Novel Wuhan (2019-nCoV) coronavirus. Am J Respir Crit Care Med. 2020;201 (4): 7-8.
12. Li Q, Guan X, Wu P, Wang X, Zhou L, Tong $Y$. Early transmission dynamics in Wuhan, China, of novel coronavirus-infected pneumonia. N Engl J Med. 2020; 382:11991207.

13. Suárez V, Suarez-Quezada M, Oros-Ruiz S, Ronquillo De Jesús E. Epidemiology of COVID-19 in Mexico: from the 27th of February to the 30th of April 2020. Epidemiología de COVID-19 en México: del 27 de febrero al 30 de abril de 2020. Rev Clin Esp. 2020;220(8):463-471.

14. Secretaria de Salud 2021. COVID-19 México. [Consultado el día 9 de diciembre 2020]. Disponible en el sitio web: https://datos.covid-19.conacyt.mx/\#COMNac

15. Ortiz-Brizuela E, Villanueva-Reza, González-Lara MF, Tamez-Torres KM, RománMontes CM, Díaz-Mejía BA, et al. Características clínicas y epidemiológicas de pacientes diagnosticados con COVID-19 en un centro de atención terciaria de la Ciudad de México: un estudio de cohorte prospectivo. Rev Invest Clin. 2020;72(3):165-77

16. World Health Organization. Vigilancia de salud pública en relación con la COVID-19, Orientaciones provisionales del 7 de agosto de 2020. [Consultado el 09 de diciembre del 2020]. Disponible en el sitio web: https://apps.who.int/iris/bitstream/handle/10 665/334000/WHO-2019-nCoV-SurveillanceGuidance-2020.7-spa.pdf 